\title{
Tai chi improves balance in Parkinson disease
}

A new report provides further evidence that tai chi improves postural control in patients with mild to moderate Parkinson disease (PD). "Tai chi training appears to reduce balance impairments ... with additional benefits of improved functional capacity and reduced falls," report Fuzhong $\mathrm{Li}$ and colleagues in the New England Journal of Medicine.

Movement impairments are a defining feature of PD and have a substantial affect on patients' functional ability and quality of life. Li and colleagues note that exercise has an important role in slowing motor deterioration in patients. Although management of PD with resistance-based exercises has shown positive results, the authors recognize that this approach is equipment-dependent and requires safety monitoring and, as such, research on alternative types of exercise was needed.

The researchers randomly assigned 195 patients with $\mathrm{PD}$, with a disease severity stage ranging from 1-4 on the Hoehn and Yahr scale, to one of three exercise interventions: a tai chi programme tailored to challenge balance and gait, resistance training, or stretching (control). Patients participated in $1-\mathrm{h}$ sessions twice weekly for 6 months. The primary outcomes included changes from baseline in maximum excursion (which assesses the limits of movements initiated by the individual as they shift their centre of gravity without falling) and directional control (a measure of movement accuracy).

At 6 months, Li et al. found that patients in the tai chi group performed significantly better than those in the other groups for both primary outcomes, suggesting reduced dyskinesia in the tai chi group. Furthermore, the tai chi group achieved better scores on the Unified Parkinson's Disease Rating Scale III and for measures of leg strength and walking velocity compared with the control group, as well as better performance in other secondary outcomes compared with both the resistance training and control groups. Importantly, at 3-month follow-up after the intervention, patients in the tai chi group had significantly fewer falls than patients in the other groups. In addition, the improvements in primary and secondary outcomes were maintained at follow-up.

The authors remark that the improved outcomes seen in patients in the tai chi group can be translated to daily activities, such as reaching forward to take objects from a cabinet, changing from a seated to

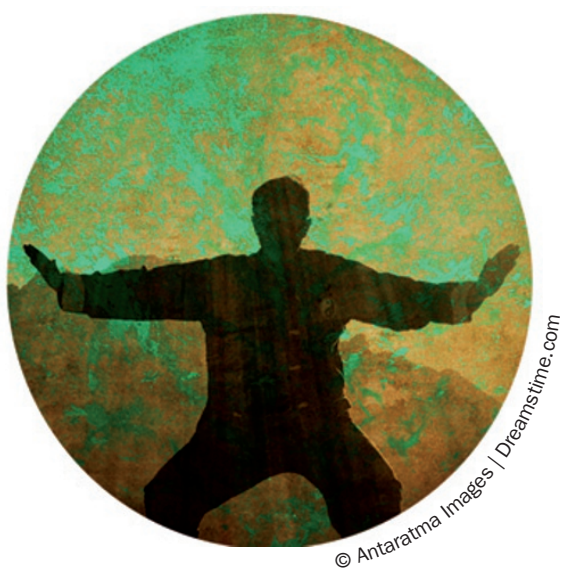

a standing position (and vice versa), and walking, while also reducing the incidence of falls. As no serious adverse events were observed during tai chi training, Li et al. conclude that this form of exercise is a safe and useful intervention for patients with $\mathrm{PD}$, and that further research is needed to determine the mechanisms involved in the improvements in motor control and mobility following tai chi.

Vanisha Parekh

Original article Li, F. et al. Tai chi and postural stability in patients with Parkinson's disease. N. Engl. J. Med. 366, 511-519 (2012) 